\title{
Investigation of three-dimensional vibration measurement by a single scanning laser Doppler vibrometer
}

\author{
Da-Ming Chen, W.D. Zhu* \\ Department of Mechanical Engineering, University of Maryland, Baltimore County, 1000 \\ Hilltop Circle, Baltimore, MD 21250, USA
}

\begin{abstract}
A scanning laser Doppler vibrometer (SLDV) has been widely used in noncontact vibration measurement. This paper presents a novel investigation of three-dimensional (3D) vibration measurement by a single SLDV sequentially placed at three different positions, where $3 \mathrm{D}$ vibration is defined as three vibration components along axes of a specified measurement coordinate system (MCS), which can give more precise knowledge of structural dynamic characteristics. A geometric model of the SLDV is proposed and a vibrometer coordinate system (VCS) based on the geometric model is defined and fixed on the SLDV. The pose of a SLDV with respect to a MCS is expressed in the form of a translation vector and a direction cosine matrix from the VCS to the MCS, which can be calculated by four or more target points with known coordinates in both the MCS and the VCS. An improved method based on the least squares method and singular value decomposition is proposed to obtain the pose of the SLDV. Compared with an inverse method, the proposed method can yield an orthogonal direction cosine matrix and be applicable to a two-dimensional (2D) structure. Effects of the number of target points on the accuracy and stability of the proposed method are investigated. With three direction cosine matrices of three different positions obtained by the proposed method, measured vibration velocities along laser line-of-sight directions can be transformed to vibration components along axes of the MCS. An experiment was conducted to measure 3D vibration
\end{abstract}

\footnotetext{
*Corresponding author. Tel: +1 410455 3394; fax: +1 4104551052.

Email addresses: damingc1@umbc.edu (Da-Ming Chen), wzhu@umbc.edu (W.D. Zhu)
} 
of a target point on a beam under sinusoidal excitation by a single SLDV sequentially placed at three different positions. Vibration components along axes of the MCS obtained by the single SLDV were in good agreement with those from a commercial Polytec 3D scanning laser vibrometer PSV-500-3D.

Keywords: 3D vibration measurement, scanning laser Doppler vibrometer, geometric model, least squares method, singular value decomposition

\section{Introduction}

A laser Doppler vibrometer (LDV) is a non-contact measurement instrument that can measure the surface velocity of a vibrating structure along the laser line-of-sight direction, using Doppler shift between an incident light and the scattered light that returns to the instrument $[1,2,3,4]$. It has a distinct advantage of measuring a lightweight structure without having to attach a transducer to it, which can locally stiffen or mass load the structure. A laser beam emitted from a LDV can be directed to any visible target point on a structure by installing a scanner in front of the LDV, which consists of a pair of orthogonal scan mirrors, and the whole system is called a scanning LDV (SLDV). This technique has greatly increased spatial resolution of vibration measurement, since the laser spot on the structure that results from the laser beam can stay at one target point long enough to acquire sufficient vibration data of that point and then move to the next one by controlling rotation angles of scan mirrors via a computer $[5,6,7]$. Angular vibration is measured in Ref. [5] by continuously applying small circular scans. Giuliani et al. [8] developed a new scanning device that can measure all six degrees of freedom at a measurement point with a LDV and a simple setup by combining various small circular scans. Yang and Allen [9] used a harmonic transfer function to process the continuous SLDV output of a downhill ski and obtain translational and rotational velocities with small circular scans.

A single SLDV is usually used to measure out-of-plane vibration of a structure surface. There is an angle between the laser beam direction and the out-of-plane direction while scanning. This angle can be neglected when the area of the measured surface is small. However, for a large scanning area and a short distance between the SLDV and the measured structure, this assumption can amplify measurement error and the out-of-plane vibration may not completely agree with the corresponding measured vibration. A cosine correction procedure is needed to obtain the accurate out-of-plane 
vibration from the measured vibration [10]. Also, a single SLDV cannot distinguish in-plane and out-of-plane vibration components when the structure has three-dimensional (3D) vibration. In order to overcome the drawback of a single SLDV, a 3D SLDV is developed and has been commercialized (e.g., Polytec PSV-400-3D or PSV-500-3D), where three laser beams coming from three SLDVs simultaneously direct to a target point and measure its velocities [11]. The measured velocities from the three SLDVs can be transformed to vibration components along axes of a specified measurement coordinate system (MCS). Many applications have been investigated using a commercial 3D SLDV. Vuye et al. [12] measured the dynamic strain field of a fan blade using a Polytec PSV-400-3D and indicated that a normal strain far below one microstrain could be measured. Weisbecker et al. [13] used 3D displacement data from a Polytec PSV-400-3D to estimate the dynamic in-plane strain over the surface of a planar structure subjected to in-plane loading. Ihrle et al. [14] used three Polytec OFV-534 LDVs to measure velocities and displacements of several points on two ossicles connected by a biological joint and reconstruct relative motion of the two ossicles. These applications show a great promise of using SLDVs for 3D vibration measurement in modern engineering. However, the cost of a commercial 3D SLDV is quite high. If a measured structure is under steady vibration, 3D vibration measurement can be achieved by a cost-effective way by sequentially placing a single SLDV at three positions [15].

With a commercial 3D SLDV or a single SLDV sequentially placed at three positions, in order to obtain vibration components along axes of a MCS, three laser beam directions at the three positions with respect to a MCS must be known. A laser beam direction with respect to its own vibrometer coordinate system (VCS) is determined by rotation angles of scan mirrors and can be obtained by the proposed geometric model of the SLDV. To obtain the direction cosine matrix from the VCS to the MCS, two methods were investigated. Zeng et al. [16] used a geometric method to obtain the matrix by the equivalent angle and equivalent axis theory. However, in this method, two direction cosine matrices with opposite sign angles are obtained and one needs to identify the correct one, which may not be easy. Xu and Miles [17] summarized an inverse method to obtain the matrix. However, the matrix obtained by this method is not guaranteed to be orthogonal. The condition number of a matrix that contains coordinates of target points in the VCS is also large when the structure is two-dimensional (2D), since there are two rows in the matrix that are almost linearly dependent. Rothberg 
and Tirabassi [18] proposed a universal framework for modeling a measured velocity in laser vibrometry and introduced its application to three common scanning vibrometer systems. Li et al. [19] developed an analytical inverse solution to obtain tilting angles of a pair of orthogonal prisms under a specified target trajectory. Inverse solutions for a Risley prism scanner with an iterative refinement by a forward solution are investigated in Ref. [20], which can quickly and accurately create a desired tracking pattern.

An improved method to calculate the direction cosine matrix is proposed in this work. Compared with the inverse method in Ref. [17], the proposed method can yield an orthogonal direction cosine matrix and be applicable to a 2D structure. Effects of the number of target points on the accuracy and stability of the proposed method are investigated. An experiment was conducted to measure $3 \mathrm{D}$ vibration of a target point on a beam under sinusoidal excitation by a single SLDV sequentially placed at three different positions. The pose of the SLDV at each position with respect to a MCS is expressed in the form of a translation vector and a direction cosine matrix from the VCS to the MCS and obtained by the proposed method. Vibration components along axes of the MCS obtained by the single SLDV sequentially placed at three different positions were in good agreement with results from a commercial Polytec 3D SLDV PSV-500-3D.

The remaining part of the paper is outlined as follows. The geometric model of a SLDV is presented in Sec. 2.1, the proposed method to obtain the pose of a SLDV with respect to a MCS is shown in Sec. 2.2 and 3D vibration calculation in a MCS is presented in Sec. 2.3. The accuracy and stability of the proposed method are investigated in Sec. 3. Experimental investigation of $3 \mathrm{D}$ vibration measurement is presented in Sec. 4. Conclusion of the work is provided in Sec. 5 .

\section{Methodology}

\subsection{Geometric model of a $S L D V$}

A single SLDV developed in this work consists of a Polytec OFV-353 LDV and a Cambridge $6240 \mathrm{H}$ scanner, as shown in Fig. 1(a). The LDV has three measurement ranges: $5 \mathrm{mms}^{-1} \mathrm{~V}^{-1}, 25 \mathrm{mms}^{-1} \mathrm{~V}^{-1}$ and $125 \mathrm{mms}^{-1} \mathrm{~V}^{-1}$. The scanner has two orthogonal scan mirrors called $\mathrm{X}$ and $\mathrm{Y}$ mirrors, which control horizontal and vertical movements of a laser spot, respectively. Rotations of scan mirrors are driven by corresponding stepper motors. The

rotation angle range of either mirror in Fig. 1(a) is $\pm 10^{\circ}$, which corresponds 
to an input voltage of $\pm 10 \mathrm{~V}$. Hence, the sensitivity of the scanner is $1^{\circ} / \mathrm{V}$. Initial positions of scan mirrors are $45^{\circ}$ with respect to the horizontal plane when the input voltages are zero.

A geometric model of the SLDV that describes the principle of scanning is shown in Fig. 1(b). There are two coordinate systems that need to be defined. The MCS $o-x y z$ is usually determined by vibration directions in which one needs to measure. The VCS $o^{\prime}-x^{\prime} y^{\prime} z^{\prime}$ is specified as follows: $x^{\prime \prime}$ and $y^{\prime}$ axes are along rotation axes of $\mathrm{X}$ and $\mathrm{Y}$ mirrors, respectively. Positive directions of the $x^{\prime \prime}$ and $y^{\prime}$ axes are directed to sides of corresponding stepper motors. The $x^{\prime}$ axis is parallel to the $x^{\prime \prime}$ axis and has an intersection point $o^{\prime}$ with the $y^{\prime}$ axis. The $z^{\prime}$ axis is perpendicular to the plane formed by the $x^{\prime}$ and $y^{\prime}$ axes; it goes through point $o^{\prime}$ and has an intersection point $o^{\prime \prime}$ with the $x^{\prime \prime}$ axis. Both the MCS and the VCS are right-handed coordinate systems. The length of $o^{\prime} o^{\prime \prime}$, denoted by $d$, is defined as the separation distance between the two mirrors, which is a constant that is specified with the scanner. Variables $\alpha$ and $\beta$ are rotation angles of $\mathrm{X}$ and $\mathrm{Y}$ mirrors measured from their initial positions, respectively; they can be indirectly obtained by corresponding input voltages to the stepper motors and the sensitivity of the scanner. Positive directions of $\alpha$ and $\beta$ are shown in Fig. $1(\mathrm{~b})$. 


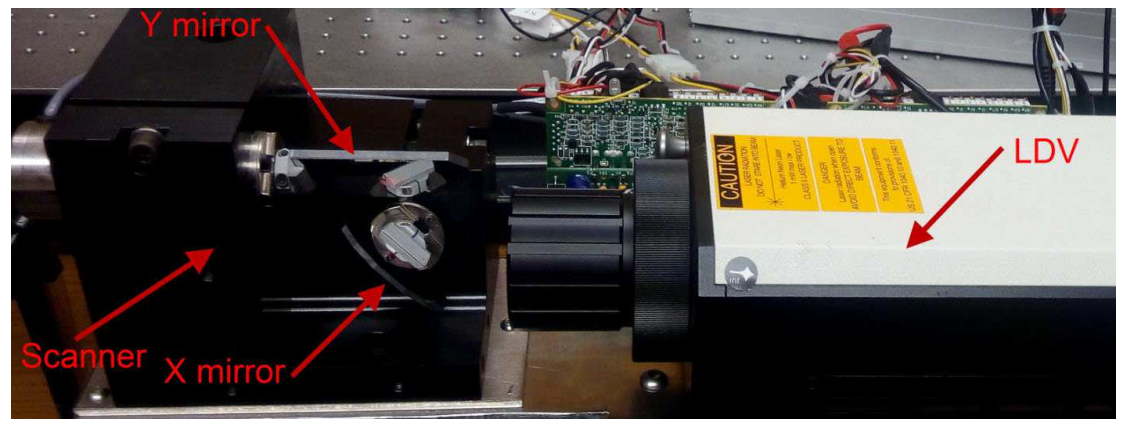

(a)

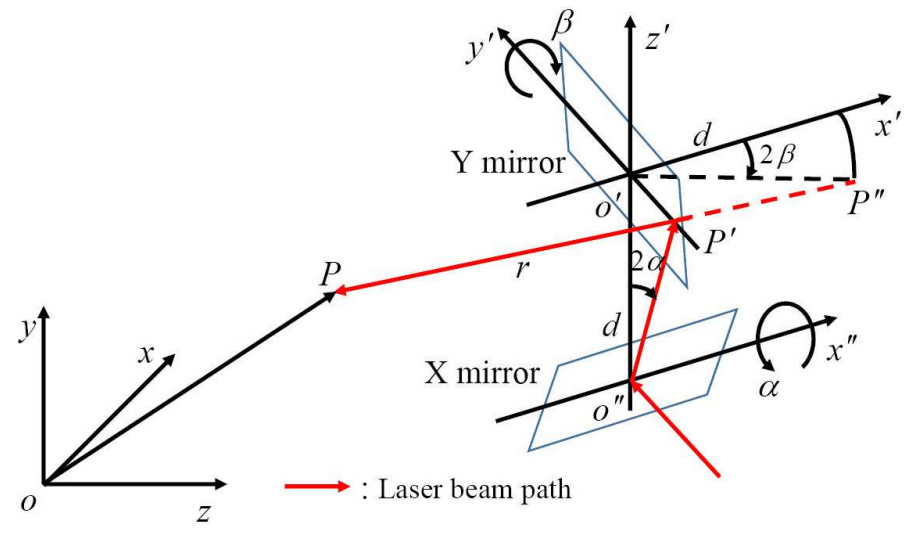

(b)

Fig. 1 (a) SLDV developed in this work and (b) a geometric model of the SLDV.

There are three fundamental operations of the scanner: (a) scan mirrors are at their initial positions, (b) only the $\mathrm{X}$ mirror rotates and the laser spot moves in the $o^{\prime} x^{\prime} y^{\prime}$ plane, and (c) only the Y mirror rotates and the laser spot moves in the $o^{\prime} x^{\prime} z^{\prime}$ plane, as shown in Fig. 2(a)-(c), respectively. One can see that each target point corresponds to a unique set of rotation angles $\alpha$ and $\beta$. From Fig. 1(b) it can be seen that $\alpha$ and $\beta$ determine positions of points $P^{\prime}$ and $P^{\prime \prime}$. Point $P^{\prime}$ is the incident point of the $\mathrm{Y}$ mirror and point $P^{\prime \prime}$ is the image point of point $o^{\prime \prime}$. Their coordinates in the VCS can be expressed as

$$
\begin{gathered}
P^{\prime}=[0,-d \tan (2 \alpha), 0]^{T} \\
P^{\prime \prime}=[d \cos (2 \beta), 0,-d \sin (2 \beta)]^{T}
\end{gathered}
$$


respectively, where the superscript $T$ denotes transpose of a vector or matrix. The line from point $P^{\prime}$ to point $P^{\prime \prime}$ is the laser beam direction and the corresponding unit direction vector in the VCS is

$$
\mathbf{e}^{\prime}=\frac{P^{\prime} P^{\prime \prime}}{\left|P^{\prime} P^{\prime \prime}\right|}=[\cos (2 \alpha) \cos (2 \beta), \sin (2 \alpha),-\cos (2 \alpha) \sin (2 \beta)]^{T}
$$

The measured velocity along the unit direction vector is

$$
\mathbf{v}^{\prime}=\left|\mathbf{v}^{\prime}\right| \mathbf{e}^{\prime}
$$

Further, coordinates of a target point $P$ in the VCS shown in Fig. 1(b) can be expressed as

$$
\begin{aligned}
P & =P^{\prime}-r \mathbf{e}^{\prime} \\
= & {[0,-d \tan (2 \alpha), 0]^{T}+[-r \cos (2 \alpha) \cos (2 \beta),-r \sin (2 \alpha), r \cos (2 \alpha) \sin (2 \beta)]^{T} } \\
& =[-r \cos (2 \alpha) \cos (2 \beta),-r \sin (2 \alpha)-d \tan (2 \alpha), r \cos (2 \alpha) \sin (2 \beta)]^{T}
\end{aligned}
$$

where $r$ is the distance from point $P^{\prime}$ to point $P$. It can be seen from Eq. (5) that the coordinates of point $P$ in the VCS are determined by variables $\alpha, \beta$, and $r$. 


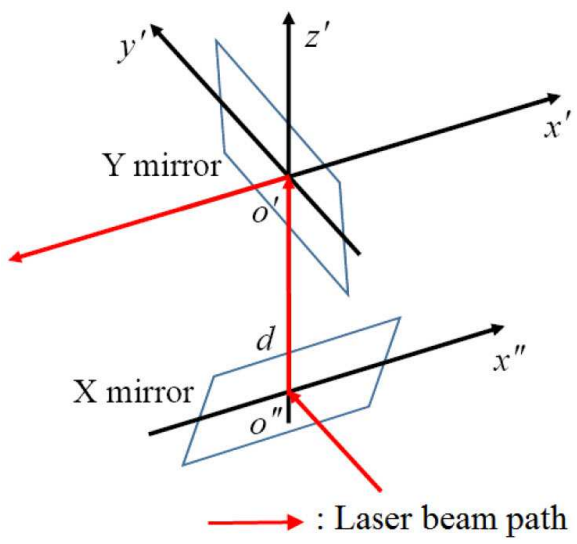

(a)

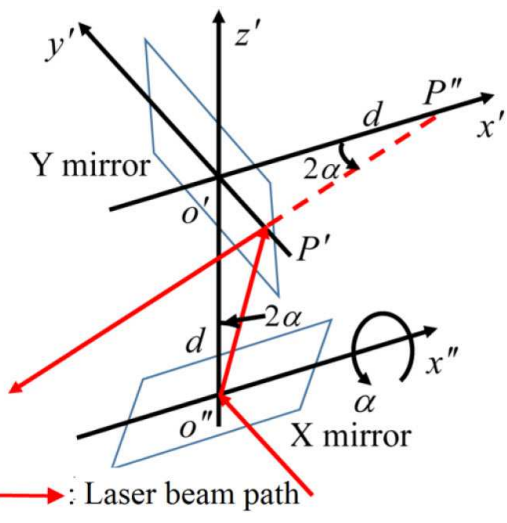

(b)

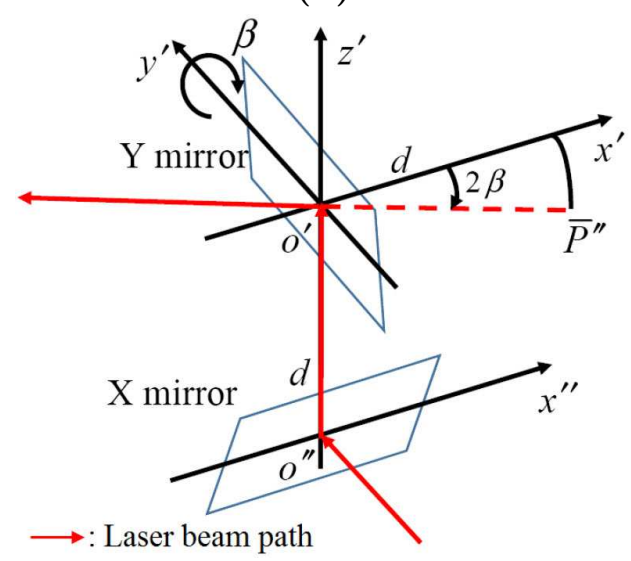

(c)

Fig. 2 (a) Scan mirrors at their initial positions, (b) only the $\mathrm{X}$ mirror rotates, and (c) only the Y mirror rotates. 


\subsection{Pose of a $S L D V$ with respect to a $M C S$}

For a target point $P_{i}(i=1, \ldots, N)$ on the surface of a measured structure, the relation of its coordinates in the MCS and the VCS can be established by

$$
\begin{aligned}
{\left[\begin{array}{l}
x_{i} \\
y_{i} \\
z_{i}
\end{array}\right]=\mathbf{T}+\mathbf{R}\left[\begin{array}{l}
x_{i}^{\prime} \\
y_{i}^{\prime} \\
z_{i}^{\prime}
\end{array}\right] } \\
=\left[\begin{array}{l}
x_{o \prime} \\
y_{o \prime} \\
z_{o \prime}
\end{array}\right]+\left[\begin{array}{lll}
\cos \left(x, x^{\prime}\right), & \cos \left(x, y^{\prime}\right), & \cos \left(x, z^{\prime}\right) \\
\cos \left(y, x^{\prime}\right), & \cos \left(y, y^{\prime}\right), & \cos \left(y, z^{\prime}\right) \\
\cos \left(z, x^{\prime}\right), & \cos \left(z, y^{\prime}\right), & \cos \left(z, z^{\prime}\right)
\end{array}\right]\left[\begin{array}{l}
x_{i}^{\prime} \\
y_{i}^{\prime} \\
z_{i}^{\prime}
\end{array}\right]
\end{aligned}
$$

where $\left[x_{i}, y_{i}, z_{i}\right]^{T}$ and $\left[\begin{array}{lll}x_{i}^{\prime}, & y_{i}^{\prime}, & z_{i}^{\prime}\end{array}\right]^{T}$ are coordinates of the target point $P_{i}$ in the MCS and the VCS, respectively, $\mathbf{T}=\left[\begin{array}{lll}x_{o \prime}, & y_{o^{\prime}}, & z_{o^{\prime}}\end{array}\right]^{T}$ is a translation vector that specifies coordinates of origin $o^{\prime}$ in the MCS, $\mathbf{R}$ is the direction cosine matrix from the VCS to the MCS and $\cos (\cdot, \cdot)$ denotes a direction cosine between two axes. The translation vector $\mathbf{T}$ together with the direction cosine matrix $\mathbf{R}$ can determine the pose of a SLDV with respect to the MCS. In order to obtain $\mathbf{T}$ and $\mathbf{R}$, coordinates of at least four target points in the MCS and the VCS must be known. The coordinates of target points in the MCS are easy to obtain and the corresponding rotation angles can also be determined by directing the laser spot to the target points. The variable $r$ in Eq. (5) can be calculated by a geometric method shown in Fig. 3. The distance between target points $P_{i}$ and $P_{j}$ remains the same when their coordinates are expressed in the MCS and the VCS, which gives

$$
\sqrt{\left(x_{i}-x_{j}\right)^{2}+\left(y_{i}-y_{j}\right)^{2}+\left(z_{i}-z_{j}\right)^{2}}=\sqrt{\left(x_{i}^{\prime}-x_{j}^{\prime}\right)^{2}+\left(y_{i}^{\prime}-y_{j}^{\prime}\right)^{2}+\left(y_{i}^{\prime}-y_{j}^{\prime}\right)^{2}}
$$

where $i$ is from 1 to $N-1$ and $j$ is an integer from $i+1$ to $N$. One can see that $N$ target points have $N$ unknown distances $r$ and can form $N \times(N-1)$ equations like Eq. (7). This is an over-determined nonlinear problem when $N \geq 4$, which can be solved by the nonlinear least squares method. The initial value of distance $r_{i}$ can be obtained by measuring the length from the Y mirror to a target point $P_{i}$, which provides a convenient way to obtain good estimates of the initial values to solve the equations. 


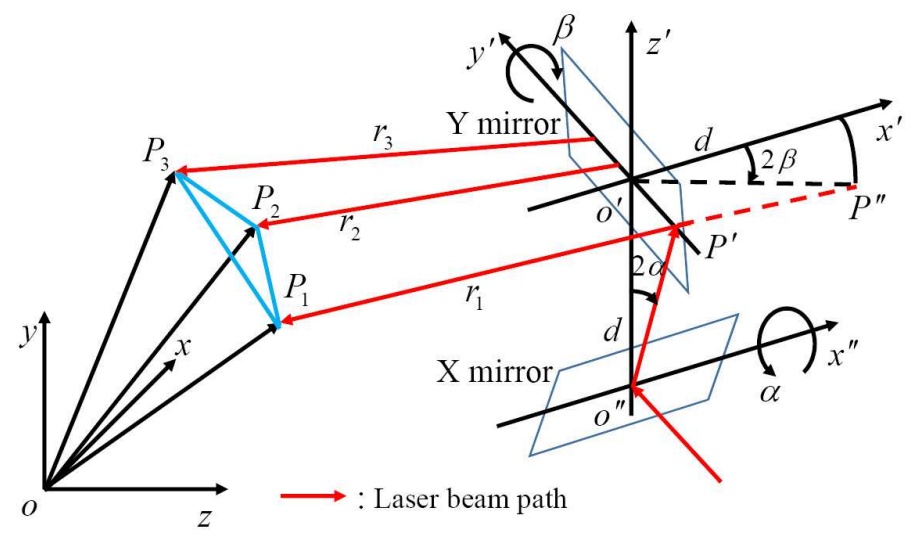

Fig. 3 Geometric method to determine the distance $r$.

In Ref. [17], coordinates of four target points in the MCS and the VCS were used to calculate $\mathbf{T}$ and $\mathbf{R}$, which are expressed as

$$
\begin{aligned}
& {[\mathbf{R}, \mathbf{T}]=\left[\begin{array}{llll}
\cos \left(x, x^{\prime}\right), & \cos \left(x, y^{\prime}\right), & \cos \left(x, z^{\prime}\right), & x_{o^{\prime}} \\
\cos \left(y, x^{\prime}\right), & \cos \left(y, y^{\prime}\right), & \cos \left(y, z^{\prime}\right), & y_{o \prime} \\
\cos \left(z, x^{\prime}\right), & \cos \left(z, y^{\prime}\right), & \cos \left(z, z^{\prime}\right), & z_{o^{\prime}}
\end{array}\right]} \\
& =\left[\begin{array}{llll}
x_{1}, & x_{2}, & x_{3}, & x_{4} \\
y_{1}, & y_{2}, & y_{3}, & y_{4} \\
z_{1}, & z_{2}, & z_{3}, & z_{4}
\end{array}\right]\left[\begin{array}{cccc}
x_{1}^{\prime}, & x_{2}^{\prime}, & x_{3}^{\prime}, & x_{4}^{\prime} \\
y_{1}^{\prime}, & y_{2}^{\prime}, & y_{3}^{\prime}, & y_{4}^{\prime} \\
z_{1}^{\prime}, & z_{2}^{\prime}, & z_{3}^{\prime}, & z_{4}^{\prime} \\
1, & 1, & 1, & 1
\end{array}\right]^{-1}
\end{aligned}
$$

However, orthogonality is an inherent property of a direction cosine matrix and one can see that the direction cosine matrix obtained by Eq. (8) cannot prove to be orthogonal. Equation (8) cannot either directly solve a case when target points are coplanar, since the second matrix on the right-hand side of Eq. (8) is almost singular [17] and the condition number of the matrix is large. With the VCS defined in this work, singularity of the matrix is caused by almost linear dependence of the first row of the matrix with its last row.

In this work, an improved method based on the least squares method and singular value decomposition is proposed to overcome the drawback of the inverse method. Optimal solutions of $\mathbf{T}$ and $\mathbf{R}$ can be obtained by solving an optimization problem [21]

$$
F(\mathbf{T}, \mathbf{R})=\delta^{2}=\min \sum_{i=1}^{N}\left\|\mathbf{p}_{i}-\left(\mathbf{T}+\mathbf{R} \mathbf{p}_{i}^{\prime}\right)\right\|^{2}
$$


where $\mathbf{p}_{i}=\left[\begin{array}{lll}x_{i}, & y_{i}, & z_{i}\end{array}\right]^{T}$ and $\mathbf{p}_{i}^{\prime}=\left[\begin{array}{lll}x_{i}^{\prime}, & y_{i}^{\prime}, & z_{i}^{\prime}\end{array}\right]^{T}$ are vectors of coordinates of a target point $P_{i}$ in the MCS and the VCS, respectively, and $\|\cdot\|$ denotes Euclidean norm of a vector. Differentiating $F$ with respect to $\mathbf{T}$ and setting it to zero yield

$$
\frac{\partial F}{\partial \mathbf{T}}=\sum_{\mathrm{i}=1}^{\mathrm{N}}-2\left[\mathbf{p}_{\mathrm{i}}-\left(\mathbf{T}+\mathbf{R} \mathbf{p}_{\mathrm{i}}^{\prime}\right)\right]=\mathbf{0}
$$

Let $\overline{\mathbf{p}}=\left(\sum_{i=1}^{N} \mathbf{p}_{i}\right) / N$ and $\overline{\mathbf{p}}^{\prime}=\left(\sum_{i=1}^{N} \mathbf{p}_{i}^{\prime}\right) / N ; \mathbf{T}$ is obtained by

$$
\mathbf{T}=\overline{\mathbf{p}}-\mathbf{R} \overline{\mathbf{p}}^{\prime}
$$

Substituting Eq. (11) into Eq. (9) yields

$$
\begin{aligned}
& \delta^{\mathbf{2}}=\min \sum_{i=1}^{N}\left\|\left(\mathbf{p}_{i}-\overline{\mathbf{p}}\right)-\mathbf{R}\left(\mathbf{p}_{i}^{\prime}-\overline{\mathbf{p}}^{\prime}\right)\right\|^{2}=\min \sum_{i=1}^{N}\left\|\mathbf{x}_{i}-\mathbf{R} \mathbf{x}_{i}^{\prime}\right\|^{2} \\
& =\min \operatorname{Tr}\left[\mathbf{X}^{T} \mathbf{X}+\mathbf{Y}^{T} \mathbf{R}^{T} \mathbf{R Y}-\mathbf{Y}^{T} \mathbf{R}^{T} \mathbf{X}-\mathbf{X}^{T} \mathbf{R Y}\right] \\
& =\min \operatorname{Tr}\left[\mathbf{X}^{T} \mathbf{X}+\mathbf{Y}^{T} \mathbf{Y}-\mathbf{Y}^{T} \mathbf{R}^{T} \mathbf{X}-\mathbf{X}^{T} \mathbf{R Y}\right]
\end{aligned}
$$

where $\mathbf{x}_{i}=\mathbf{p}_{i}-\overline{\mathbf{p}}, \mathbf{x}_{i}^{\prime}=\mathbf{p}_{i}^{\prime}-\overline{\mathbf{p}}^{\prime}, \mathbf{X}=\left[\mathbf{x}_{1}, \mathbf{x}_{2,} \ldots, \mathbf{x}_{N}\right]$ and $\mathbf{Y}=\left[\mathbf{y}_{1}, \mathbf{y}_{2}, \ldots, \mathbf{y}_{N}\right]$ are two $3 \times N$ matrices that consist of coordinates of $N$ target points in the MCS and the VCS, respectively, and Tr denotes the trace of a matrix. Since the first two terms $\mathbf{X}^{T} \mathbf{X}$ and $\mathbf{Y}^{T} \mathbf{Y}$ on the right-hand side of Eq. (12) do not depend on $\mathbf{R}$, excluding them would not affect the optimization problem, which yields

$$
\begin{gathered}
\min \operatorname{Tr}\left[-\mathbf{Y}^{T} \mathbf{R}^{T} \mathbf{X}-\mathbf{X}^{T} \mathbf{R Y}\right]=\min \operatorname{Tr}\left[-2 \mathbf{X}^{T} \mathbf{R Y}\right]= \\
\min \operatorname{Tr}\left[-2 \mathbf{R} \mathbf{Y} \mathbf{X}^{T}\right]=\max \operatorname{Tr}\left[2 \mathbf{R} \mathbf{Y} \mathbf{X}^{T}\right]=\max \operatorname{Tr}[2 \mathbf{R} \mathbf{A}]
\end{gathered}
$$

where $\mathbf{A}=\mathbf{Y X}^{T}$ is a $3 \times 3$ matrix. With singular value decomposition, $\mathbf{A}$ can be decomposed as

$$
\mathbf{A}=\mathbf{U D V}^{T}
$$

where $\mathbf{U}$ is a $3 \times 3$ orthogonal matrix, $\mathbf{D}$ is a $3 \times 3$ diagonal matrix with non-negative real values in the descending order on the diagonal, and $\mathbf{V}$ is a $3 \times 3$ orthogonal matrix. Substituting Eq. (14) into Eq. (13) yields

$$
\begin{aligned}
\max \operatorname{Tr}\left[2 R \mathbf{U D V}^{T}\right] & =\max \operatorname{Tr}\left[2 D V^{T} R U\right] \\
& =\max \operatorname{Tr}[2 \mathbf{D M}]=\max \sum_{i=1}^{3} 2 \lambda_{i} M_{i i} \leq \sum_{i=1}^{3} 2 \lambda_{i}
\end{aligned}
$$


where $\mathbf{M}=\mathbf{V}^{T} \mathbf{R} \mathbf{U}$ is an orthogonal matrix since $\mathbf{V}, \mathbf{R}$, and $\mathbf{U}$ are all orthogonal matrices, $\lambda_{i}$ is the $i$ th non-negative singular value of $\mathbf{A}$ and $M_{i i}$ is the $i$ th diagonal element of $\mathbf{M}$. The optimization problem in Eq. (15) attains a maximum when $M_{i i}=1$. Since $\mathbf{M}$ is an orthogonal matrix, this means that $\mathbf{M}$ has to be an identity matrix. Hence, $\mathbf{R}$ is obtained by

$$
\mathbf{R}=\mathbf{V I U} \mathbf{U}^{T}=\mathbf{V U}^{T}
$$

From the definition of $\mathbf{R}$ in Eq. (6), one can see that $\mathbf{R}$ is a rotation matrix between two coordinate systems, whose determinant is 1 . If the determinant of $\mathbf{R}$ derived from Eq. (16) is $-1, \mathbf{R}$ is a reflection matrix rather than a rotation matrix [21]. If $\mathbf{R}$ is a reflection matrix when $M_{i i}=1$, one needs to find the next maximum solution to get a rotation matrix. The objective function in Eq. (15) is a linear function of variables $M_{11}, M_{22}$, and $M_{33}$. Since the domain of each variable is $[-1,1]$, the objective function attains its extremum on boundaries of the domains. If $\mathbf{R}$ is a reflection matrix when $M_{i i}=1$, the next maximum solution is $M_{11}=1, M_{22}=1$, and $M_{33}=-1$ since $\lambda_{1}>\lambda_{2}>\lambda_{3} \geq 0$. In this case, $\mathbf{R}$ is given by

$$
\mathbf{R}=\mathbf{V}\left[\begin{array}{ccc}
1, & 0, & 0 \\
0, & 1, & 0 \\
0, & 0, & -1
\end{array}\right] \mathbf{U}^{T}
$$

A general formula is obtained to calculate $\mathbf{R}$, which is applicable for both cases with the determinant of $\mathbf{V U}^{T}$ being 1 or -1 :

$$
\mathbf{R}=\mathbf{V}\left[\begin{array}{ccc}
1, & 0, & 0 \\
0, & 1, & 0 \\
0, & 0, & \operatorname{det}\left(\mathbf{V} \mathbf{U}^{T}\right)
\end{array}\right] \mathbf{U}^{T}
$$

where det denotes the determinant of a square matrix.

It can be seen from Eq. (18) that the direction cosine matrix $\mathbf{R}$ obtained by the proposed method is orthogonal, since it is the product of three orthogonal matrices. When target points are coplanar, A in Eq. (14) can still be decomposed by singular value decomposition. Hence, the proposed method can be used to calculate the pose of a SLDV with respect to a $2 \mathrm{D}$ structure, which makes it more general. The procedure to calculate the pose of a SLDV with respect to a MCS is summarized as follows:

(1) Select at least four target points whose coordinates in the MCS are known. Control scan mirrors to move the laser spot to the target points and record 
corresponding input voltages. Errors occur when determining input voltages to scan mirrors for target points, since whether the laser spot completely aligns with target points or not is determined by visual ability of an experimentalist. Then measure initial distances from the $\mathrm{Y}$ mirror to the target points.

(2) Use Eq. (5) to calculate initial coordinates of each target point in the VCS. Obtain the exact distance $r$ of each target point by solving the equations formed by Eq. (7), and calculate exact coordinates of all the target points in the VCS.

(3) Calculate $\mathbf{R}$ and $\mathbf{T}$ using Eqs. (18) and (11), respectively.

The key to calculate the pose of a SLDV with respect to a MCS is to obtain exact coordinates of target points in the VCS. It can be seen from Eq. (5) that coordinates of a target point in the VCS are determined by variables $\alpha, \beta$, and $r$. Values of $\alpha$ and $\beta$ can be accurately obtained from input voltages to the $\mathrm{X}$ and $\mathrm{Y}$ mirrors and the sensitivity of the scanner. Since $r$ is calculated by the nonlinear least squares method, it is important to obtain a good initial value for it to solve over-determined nonlinear equations. A laser measurement meter can be used to measure the initial value of $r$.

\subsection{Calculation of $3 D$ vibration in a MCS}

The laser beam direction in the VCS when measuring a target point can be determined by Eq. (3) and the direction cosine matrix $\mathbf{R}$ from the VCS to the MCS can be obtained using the proposed method in Sec. 2.2. Hence, the laser beam direction in the MCS can be expressed as

$$
\mathbf{e}=\mathbf{R e}^{\prime}
$$

If a measured structure is under steady vibration, 3D vibration measurement can be achieved by a single SLDV sequentially placed at three positions, as shown in Fig. 4. The excitation signal is the reference signal to trigger the start of the vibration measurement at each position. The relation between measured velocities and velocity components along axes of the MCS is

$$
\left[\begin{array}{l}
v_{1} \\
v_{2} \\
v_{3}
\end{array}\right]=\left[\begin{array}{l}
\mathbf{e}_{1}^{T} \\
\mathbf{e}_{2}^{T} \\
\mathbf{e}_{3}^{T}
\end{array}\right]\left[\begin{array}{l}
v_{x} \\
v_{y} \\
v_{z}
\end{array}\right]=\left[\begin{array}{lll}
\cos \left(v_{1}, x\right), & \cos \left(v_{1}, y\right), & \cos \left(v_{1}, z\right) \\
\cos \left(v_{2}, x\right), & \cos \left(v_{2}, y\right), & \cos \left(v_{2}, z\right) \\
\cos \left(v_{3}, x\right), & \cos \left(v_{3}, y\right), & \cos \left(v_{3}, z\right)
\end{array}\right]\left[\begin{array}{l}
v_{x} \\
v_{y} \\
v_{z}
\end{array}\right]
$$


where $v_{1}, v_{2}$, and $v_{3}$ are the measured velocities at the three positions, respectively, and $\mathbf{e}_{1}, \mathbf{e}_{2}$, and $\mathbf{e}_{3}$ are unit vectors of laser beam directions with respect to the MCS at the three positions, respectively. The velocity components along axes of the MCS can be calculated by

$$
\left[\begin{array}{l}
v_{x} \\
v_{y} \\
v_{z}
\end{array}\right]=\left[\begin{array}{lll}
\cos \left(v_{1}, x\right), & \cos \left(v_{1}, y\right), & \cos \left(v_{1}, z\right) \\
\cos \left(v_{2}, x\right), & \cos \left(v_{2}, y\right), & \cos \left(v_{2}, z\right) \\
\cos \left(v_{3}, x\right), & \cos \left(v_{3}, y\right), & \cos \left(v_{3}, z\right)
\end{array}\right]^{-1}\left[\begin{array}{l}
v_{1} \\
v_{2} \\
v_{3}
\end{array}\right]
$$

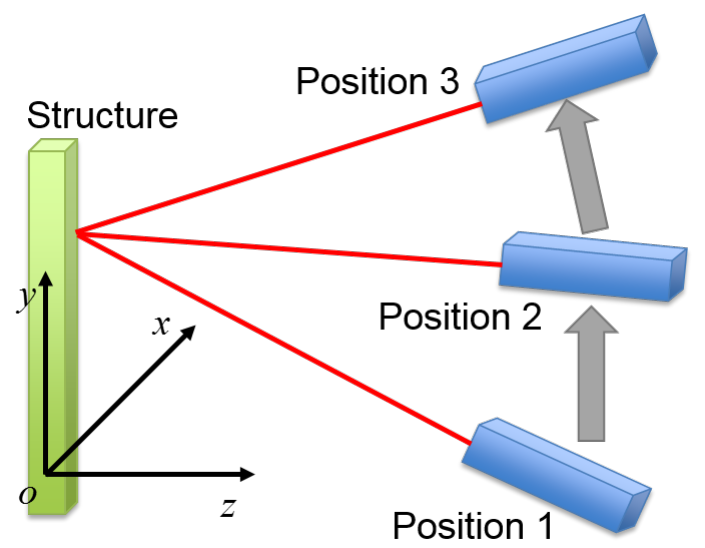

Fig. 4 3D vibration measurement by a single SLDV.

The procedure to calculate $3 \mathrm{D}$ vibration in a MCS is summarized as follows:

(1) Define a MCS for a vibrating structure and 3D vibration of the structure is calculated in this coordinate system. Coordinates of at least four target points in this coordinate system need to be known.

(2) Place a single SLDV at position 1. The pose of the VCS with respect to the MCS is fixed. Apply the proposed method described in Sec. 2.2 to obtain $\mathbf{R}_{\mathbf{1}}$ and $\mathbf{T}_{\mathbf{1}}$ from the VCS to the MCS.

(3) Control scan mirrors to move the laser spot to a measurement point on the vibrating structure and measure vibration along the laser line-of-sight direction.

(4) Move the single SLDV to position 2 and repeat steps (2) and (3).

(5) Move the single SLDV to position 3 and repeat steps (2) and (3).

(6) Calculate 3D vibration in the MCS from measured vibration by Eq. (21). Measurement at each position is independent and does not affect one another. 


\section{Investigation of accuracy and stability of the proposed method}

In order to investigate the accuracy and stability of the proposed method, an experiment was conducted to calculate the pose of a SLDV with respect to a MCS, as shown in Fig. 5(a). A Polytec PSV-A-450 reference object was used to provide accurate coordinates of target points, as shown in Fig. 5(b). Two coordinate systems MCS and VCS were defined, which were fixed on the reference object and the SLDV, respectively. The SLDV in Fig. 1(a) was placed at a position to have a large laser beam angle with the $z$ axis of the MCS. Rotation angles of scan mirrors were controlled by a dSPACE 1103 controller board. Twelve target points were selected; their coordinates in the MCS and corresponding input voltages and initial distances are listed in Table 1. 


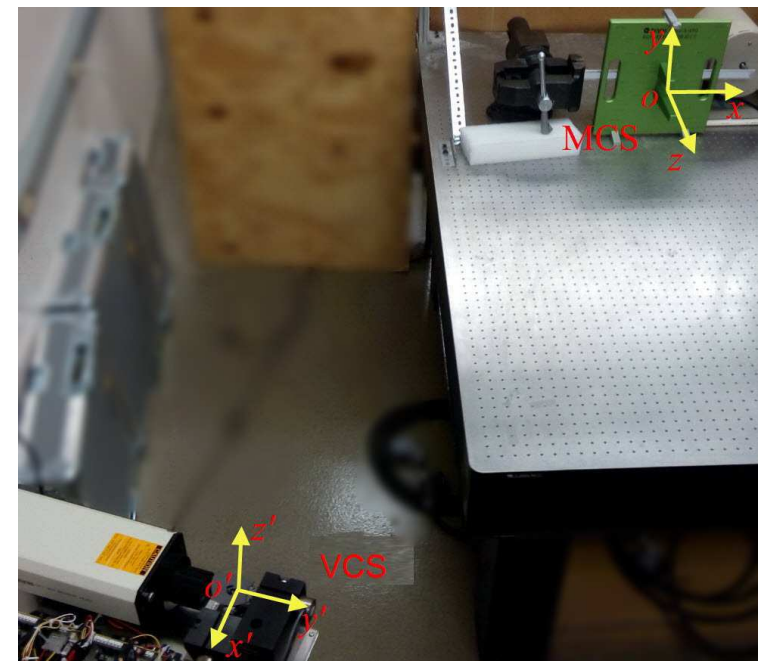

(a)

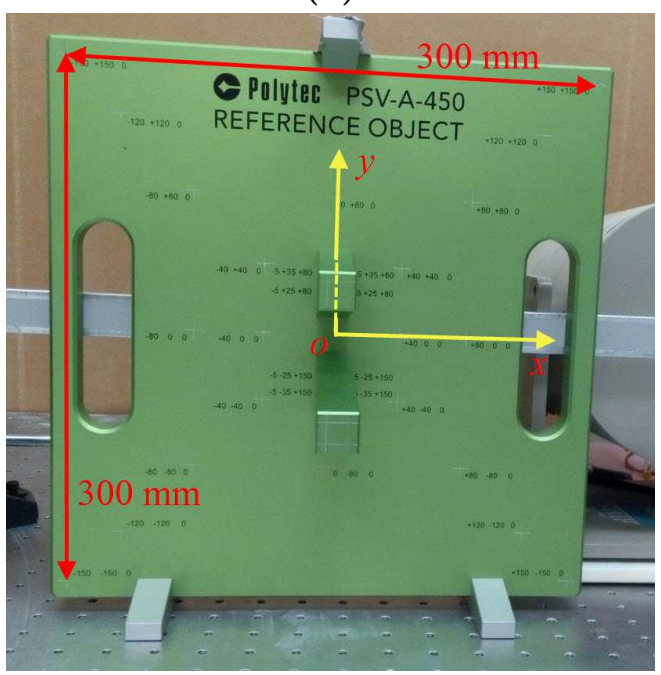

(b)

Fig. 5 (a) Experimental setup to calculate the pose of a SLDV with respect to a MCS and (b) a Polytec PSV-A-450 reference object.

\subsection{Comparison between the inverse and proposed methods}

In order to compare the proposed method with the inverse method, four target points in Table 1 with indices 1, 2, 5, and 9 were selected to calculate the pose of the SLDV with respect to the MCS. The four target points should 
Tab. 1 Coordinates of target points in the MCS and corresponding input voltages and initial distances

\begin{tabular}{ccccc}
\hline Index & Coordinates $(\mathrm{mm})$ & $\mathrm{X}$ mirror $(\mathrm{V})$ & $\mathrm{Y}$ mirror $(\mathrm{V})$ & $r_{0}(\mathrm{~mm})$ \\
\hline 1 & $(150,150,0)$ & -7.395 & 1.690 & 1900 \\
2 & $(150,-150,0)$ & -7.388 & -2.950 & 1900 \\
3 & $(-150,-150,0)$ & -3.515 & -3.195 & 1900 \\
4 & $(-150,150,0)$ & -3.512 & 1.747 & 1900 \\
5 & $(5,35,80)$ & -6.337 & -0.130 & 1770 \\
6 & $(5,25,80)$ & -6.337 & -0.300 & 1770 \\
7 & $(-5,25,80)$ & -6.205 & -0.300 & 1770 \\
8 & $(-5,35,80)$ & -6.205 & -0.140 & 1770 \\
9 & $(5,-25,150)$ & -7.010 & -1.190 & 1720 \\
10 & $(5,-35,150)$ & -7.010 & -1.357 & 1720 \\
11 & $(-5,-35,150)$ & -6.873 & -1.363 & 1720 \\
12 & $(-5,-25,150)$ & -6.873 & -1.191 & 1720 \\
\hline
\end{tabular}

not be coplanar when using the inverse method. Translation vectors $\mathbf{T}$ and direction cosine matrices $\mathbf{R}$ obtained by the two methods are listed in Tables 2 and 3 , respectively. In Table 2 , the translation vectors obtained by the two methods, which matched with the experimental setup, were close to each other. In Table 3, the direction cosine matrices from the two methods, which matched with the relation between the MCS and the VCS, were also close to each other. However, the element $\cos \left(y, z^{\prime}\right)$ from the inverse method was 1.0006 and larger than one, which violated the definition of a direction cosine. Also, Euclidean norm of the second column was 0.9885 and not equal to one, which violated a mathematical property of a direction cosine matrix. The results from the inverse method show that though it can obtain the pose of the SLDV and may meet an engineering need, it cannot obtain the direction cosine matrix that satisfies all the mathematical properties of a direction cosine matrix. On the other hand, the direction cosine matrix obtained by the proposed method can satisfy all the mathematical properties of a direction cosine matrix, as shown in Table 3.

With the proposed method, an error $\delta_{i}$ for each target point can be calculated to evaluate the accuracy of $\mathbf{T}$ and $\mathbf{R}$ :

$$
\delta_{i}=\left\|\mathbf{p}_{i}-\left(\mathbf{T}+\mathbf{R} \mathbf{p}_{i}^{\prime}\right)\right\|
$$


Tab. 2 Translation vectors $\mathbf{T}$ from the two methods

\begin{tabular}{cccc}
\hline Unit: mm & $x$ & $y$ & $z$ \\
\hline Inverse method & -1018.2 & 66.7 & 1514.3 \\
Proposed method & -1013.7 & 63.6 & 1515.5 \\
\hline
\end{tabular}

Tab. 3 Direction cosine matrices $\mathbf{R}$ from the two methods

\begin{tabular}{ccccc}
\hline & & $x^{\prime}$ & $y^{\prime}$ & $z^{\prime}$ \\
\hline \multirow{3}{*}{ Inverse method } & $x$ & -0.3869 & 0.9133 & 0.0103 \\
& $y$ & 0.0140 & 0.0003 & 1.0006 \\
& $z$ & 0.9227 & 0.3782 & -0.0069 \\
\hline \multirow{3}{*}{ Proposed method } & $x$ & -0.3815 & 0.9243 & 0.0105 \\
& $y$ & 0.0103 & -0.0071 & 0.9999 \\
& $z$ & 0.9243 & 0.3816 & -0.0069 \\
\hline
\end{tabular}

In this experiment, errors for the four target points with indices 1, 2, 5, and 9 are $173 \mu \mathrm{m}, 79 \mu \mathrm{m}, 466 \mu \mathrm{m}$, and $309 \mu \mathrm{m}$, respectively. The stand-off distance from the VCS to the MCS was $1824 \mathrm{~mm}$, which corresponded to a laser spot size of $148 \mu \mathrm{m}$. The errors and the laser spot size were of the same order, which shows that $\mathbf{T}$ and $\mathbf{R}$ obtained by the proposed method can accurately indicate the pose of the SLDV with respect to the MCS.

\subsection{Effects of the number of target points}

In order to investigate the stability of the proposed method, the number of target points from four to 12 was selected from Table 1 to calculate $\mathbf{T}$ and R. Indices of each group are $(1,2,5,9),(1,2,5,6,9),(1,2,5,6,9,10),(1$, $2,3,5,6,9,10),(1,2,3,5,6,7,9,10),(1,2,3,5,6,7,9,10,11),(1,2,3$, $4,5,6,7,9,10,11),(1,2,3,4,5,6,7,8,9,10,11)$, and $(1,2,3,4,5,6$, $7,8,9,10,11,12)$. The value of each element in the direction cosine matrix with different numbers of target points is shown in Fig. 6(a). Corresponding mean errors and standard deviations are calculated by

$$
\begin{gathered}
\bar{\delta}_{N}=\left(\sum_{i=1}^{N} \delta_{N i}\right) / N \\
s_{N}=\sqrt{\frac{\sum\left(\delta_{N i}-\bar{\delta}_{N}\right)^{2}}{N-1}}
\end{gathered}
$$


respectively, where $N$ is from four to 12 . Results of $\bar{\delta}_{N}$ and $s_{N}$ with different numbers of target points are shown in Fig. 6(b). One can see that there is little change of all the elements in $\mathbf{R}$ with different numbers of target points, which indicates that the proposed method is stable and not sensitive to the number of target points selected to calculate the pose of the SLDV. Ranges of mean errors and standard deviations are $[257,634] \mu \mathrm{m}$ and $[111,415] \mu \mathrm{m}$, respectively. They have the same order as the laser spot size. The small mean errors and standard deviations show that the number of target points does not affect much the accuracy and stability of $\mathbf{T}$ and $\mathbf{R}$.

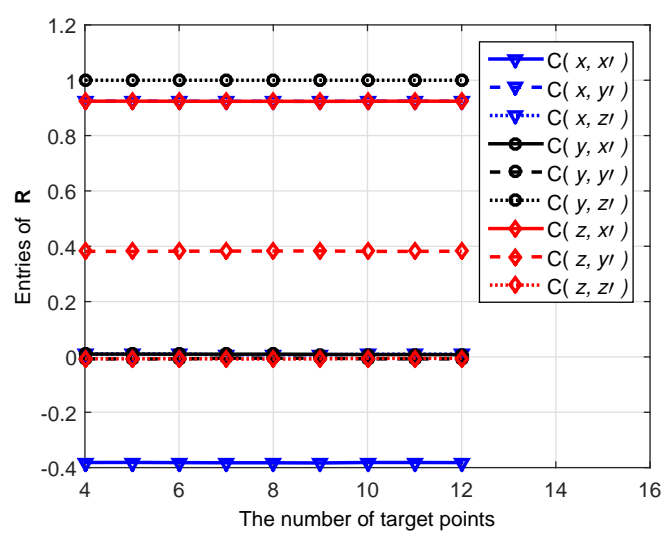

(a)

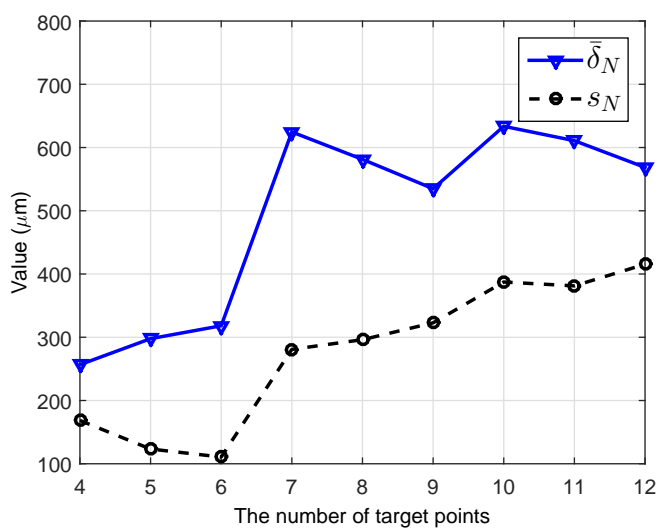

(b)

Fig. 6 (a) Elements of $\mathbf{R}$ with different numbers of target points and (b) mean errors $\bar{\delta}_{N}$ and standard deviations $s_{N}$ with different numbers of target points. 
Tab. 4 Second matrix on the right-hand side of Eq. (8) when target points are coplanar

\begin{tabular}{cccc}
\hline-1843.0 & -1846.6 & -1736.3 & -1731.6 \\
\hline 496.5 & 498.8 & 219.7 & 218.0 \\
\hline 108.8 & -190.8 & -194.5 & 105.7 \\
\hline 1 & 1 & 1 & 1 \\
\hline
\end{tabular}

Tab. 5 Translation vectors $\mathbf{T}$ from the two methods with use of coplanar target points

\begin{tabular}{cccc}
\hline Unit: mm & $x$ & $y$ & $z$ \\
\hline Inverse method & -1039.0 & -56.698 & 0 \\
\hline Proposed method & -993.4 & 70.0 & 1529.8 \\
\hline
\end{tabular}

\subsection{Use of the proposed method for a $2 D$ structure}

In order to compare the inverse and proposed methods when target points are coplanar, four target points with indices 1, 2, 3, and 4 were selected. The second matrix on the right-hand side of Eq. (8) in this case is listed in Table 4. One can see that the first row is almost linearly dependent with the last row and the condition number of the matrix is $8.97 \times 10^{6}$. Results of $\mathbf{T}$ and $\mathbf{R}$ from the inverse method are listed in Tables 5 and 6 , respectively; they show that the inverse method cannot be directly used due to the large condition number of the matrix. On the other hand, the proposed method does not take the inverse of the matrix and $\mathbf{A}$ in Eq. (14) can be decomposed even when all target points are coplanar. Hence, the proposed method can be used to calculate the pose of a SLDV with respect to a $2 \mathrm{D}$ structure. Results of $\mathbf{T}$ and $\mathbf{R}$ from the proposed method are listed in Tables 5 and 6 , respectively. Results from the proposed method agree well with those for the non-coplanar case in Sec. 3.1. The error $\delta_{i}$ of each target point in this case is $346 \mu \mathrm{m}$, $347 \mu \mathrm{m}, 332 \mu \mathrm{m}$, and $330 \mu \mathrm{m}$, respectively, which shows that the proposed method can accurately obtain the pose of the SLDV with respect to a 2D structure.

\section{Experimental investigation of 3D vibration measurement}

In order to investigate feasibility of $3 \mathrm{D}$ vibration measurement using a single SLDV, an experiment was conducted to measure vibration of a mea- 
Tab. 6 Direction cosine matrices $\mathbf{R}$ from the two methods with use of coplanar target points

\begin{tabular}{ccccc}
\hline & & $x^{\prime}$ & $y^{\prime}$ & $z^{\prime}$ \\
\hline \multirow{3}{*}{ Inverse method } & $x$ & -0.3971 & 0.9180 & 0.0116 \\
& $y$ & -0.3724 & -0.160 & 1.0044 \\
& $z$ & 0 & 0 & 0 \\
\hline \multirow{3}{*}{ Proposed method } & $x$ & -0.3694 & 0.9292 & 0.0112 \\
& $y$ & 0.0140 & -0.0065 & 0.9999 \\
& $z$ & 0.9292 & 0.3695 & -0.0106 \\
\hline
\end{tabular}

surement point on a beam, as shown in Fig. 7. A bench vice was used to clamp the left end of the beam to simulate a fixed boundary. A strip of retroreflective tape was attached on the surface of the beam to enhance laser reflection that directly determined the signal-to-noise ratio (SNR) of the SLDV velocity output. A MB Dynamics MODAL-50 shaker was connected to the right end of the beam with a shaker screw. The dSPACE 1103 controller board was used for data acquisition. The beam was under sinusoidal excitation by the shaker with a frequency of $110 \mathrm{~Hz}$, which was close to the first natural frequency of the beam. The reason to select this excitation frequency was that vibration of the beam excited by the shaker could have a relatively large amplitude. Each vibration component in a MCS could then have a suitable amplitude when vibration of the beam was decomposed in the MCS. The single SLDV in Fig. 1(a) was sequentially placed at three different positions to measure velocities of the same point, as shown in Fig. 8(a)-(c). In order to have the same phase for the measured velocities at the three positions, the excitation signal was used as the reference signal to trigger the start of data acquisition. The sampling frequency was $25000 \mathrm{~Hz}$ in this experiment. A MCS was defined on the reference object that was used in Sec. 3. Since the vibration direction of the beam was along the axis of the shaker, in order to let velocity components along $x$ and $z$ axes of the MCS have sufficient vibration amplitudes, the reference object was placed at an angle with the beam, as shown in Fig. 7. A top view of the three positions of the single SLDV with respect to the origin of the MCS is shown in Fig. $8(\mathrm{~d})$. 


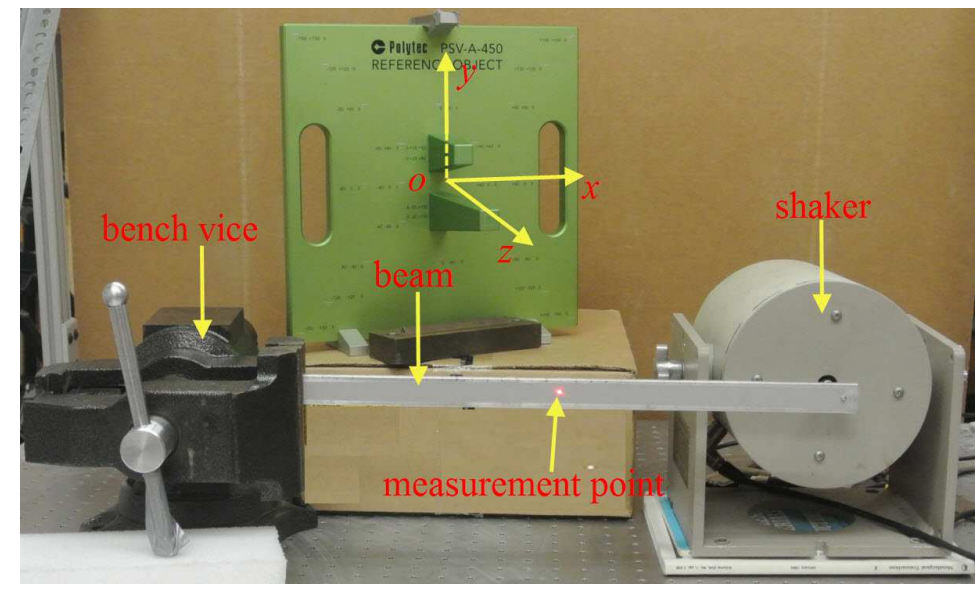

Fig. 7 Experimental setup of 3D vibration measurement. 


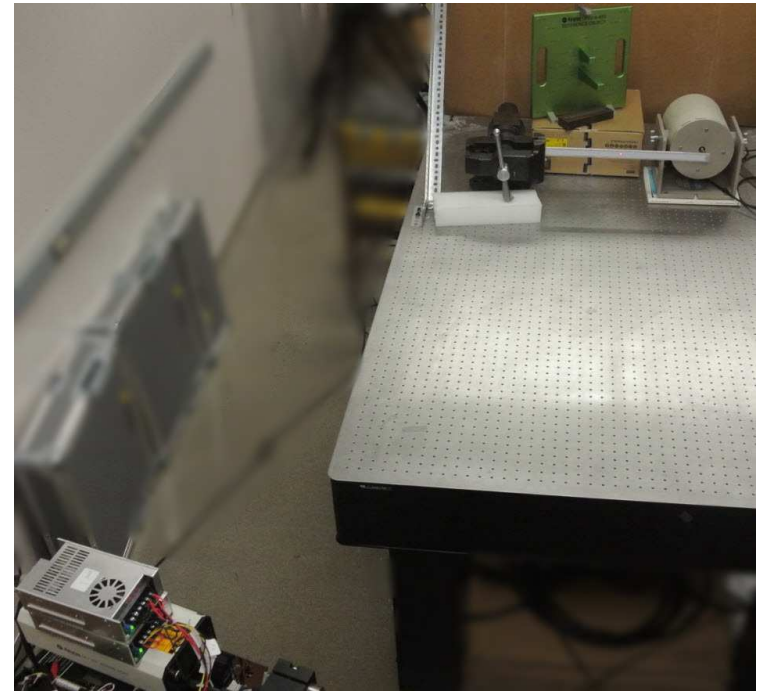

(a)

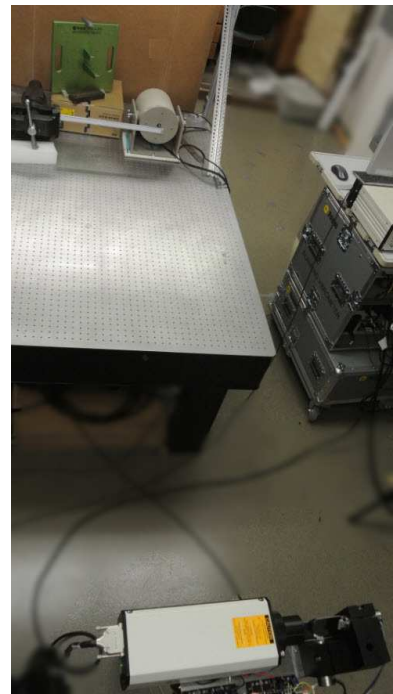

(b)

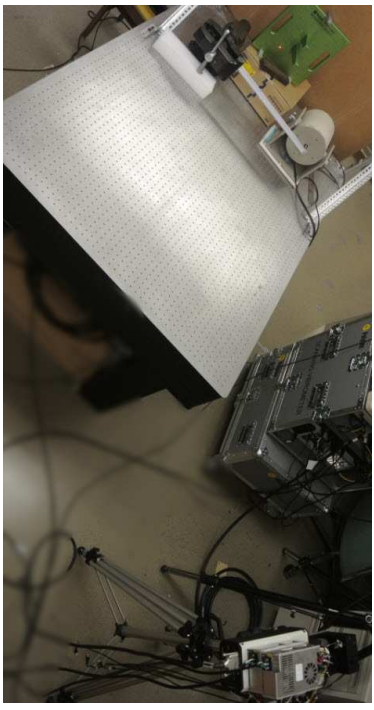

(c)

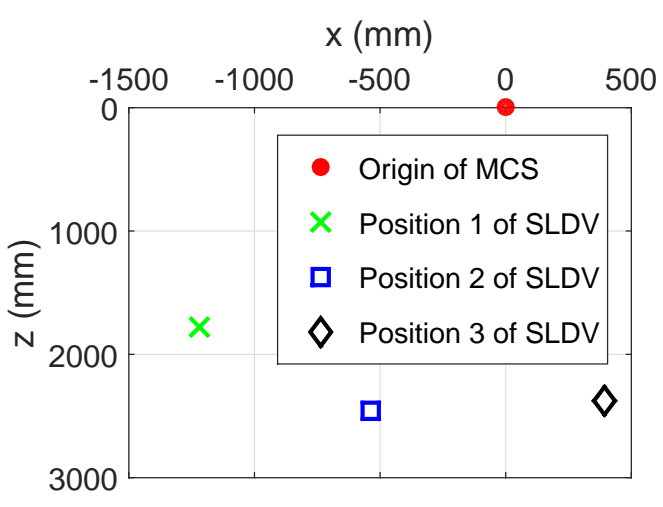

(d)

Fig. 8 The single SLDV sequentially placed at three positions: (a) position 1, (b) position 2, and (c) position 3 ; (d) a top view of the three positions of the single SLDV with respect to the origin of the MCS.

Six target points were selected to calculate the pose of the SLDV at the three positions. Their coordinates in the MCS and corresponding input voltages and initial distances are listed in Tables 7-9. Two different target points 
$(80,-80,0)$ and $(-80,-80,0)$ were used at position 3 because the laser spot could not reach $(120,-120,0)$ and $(-120,-120,0)$ at that position due to presence of the shaker in the way. Translation vectors and direction cosine matrices of the SLDV from the proposed method corresponding to the three positions are listed in Tables 10 and 11, respectively. It could be seen that the pose of the SLDV at each position matched with the experimental setup. The laser spot was then directed to the measurement point on the beam at each position. Measured velocities $v_{1}, v_{2}$, and $v_{3}$ at the three positions are shown in Fig. 9(a). Velocity components $v_{x}, v_{y}$, and $v_{z}$ axes of the MCS were obtained by Eq. (21), as shown in Fig. 9(b). One can see that amplitudes of the velocity components along the $y$ and $z$ axes were minimum and maximum, respectively, which matched with the experimental setup. In order to compare the results with those from a commercial 3D SLDV, 3D vibration of the same measurement point was measured by a Polytec PSV500-3D, as shown in Fig 10. Reasons why a tri-axial accelerometer was not used for comparison are as follows: (1) a SLDV measures a velocity while an accelerometer measures an acceleration. In order to compare measurement results, an acceleration needs to be numerically integrated to a velocity. Numerical integration has a truncation error; it may be not easy to distinguish whether errors come from measurement or numerical integration. (2) A triaxial accelerometer PCB T356A17 can have some mass loading $(9.3 \mathrm{~g})$ to the beam with a mass of $219 \mathrm{~g}$. If an accelerometer is attached at the position of the measurement point shown in Fig. 7, a SLDV cannot reach that point and can only measure the vibration of the surface on the accelerometer. If the accelerometer is attached on the back side of the measurement point, a SLDV can only measure a corresponding point on the front side of the measurement point. In either case, the accelerometer and the SLDV cannot measure the vibration of the same point on the beam. (3) The most critical reason is that a MCS is already fixed on a tri-axial accelerometer, as shown in Fig. 11. When the tri-axial accelerometer is attached on the beam, 3D vibration is measured in this MCS. For a single SLDV placed at an arbitrary position, it would be impossible to determine the angle of the laser line-of-sight direction with respect to this MCS, since there are no target points with known coordinates in this MCS and the proposed method in Section 2.2 cannot be applied. Comparison of velocity components in the MCS using a single SLDV and a PSV-500-3D is shown in Fig. 12(a)-(c). The $x$ components from the two measurement methods were in excellent agreement, and so were the $z$ components. Amplitudes of velocity components 
Tab. 7 Coordinates of target points in the MCS and corresponding input voltages and initial distances at position 1

\begin{tabular}{cccc}
\hline Coordinates $(\mathrm{mm})$ & $\mathrm{X}$ motor $(\mathrm{V})$ & $\mathrm{Y}$ motor $(\mathrm{V})$ & $r_{0}(\mathrm{~mm})$ \\
\hline$(150,150,0)$ & 0.420 & 3.210 & 2260 \\
$(120,-120,0)$ & 0.605 & -0.195 & 2260 \\
$(-120,-120,0)$ & 3.200 & -0.215 & 2120 \\
$(-150,150,0)$ & 3.635 & 3.470 & 2120 \\
$(-5,35,80)$ & 1.370 & 1.950 & 2120 \\
$(5,-35,150)$ & 0.660 & 1.080 & 2050 \\
\hline
\end{tabular}

in the MCS from the single SLDV and the Polytec PSV-500-3D are listed in Table 12. It can be seen from Table 12 that the vibration amplitudes of $y$ components were much smaller than those of the $x$ and $z$ components in the two measurement methods, as expected from the experimental setup in Fig. 7 where the $y$ axis of the MCS was almost perpendicular to the axis of the shaker. Hence, inherent noise from hardware would have more effect on the $y$ components, which makes the SNR of $y$ components was not as high as those of the $x$ and $z$ components for both the single SLDV and Polytec PSV-500-3D measurements and leads a relatively large error. One can see from Fig. 12(b) that the velocity of $y$ component from the single SLDV was a little noisy and that from the 3D SLDV had some dropouts and spikes at some instants. However, they were still in fairly good agreement. Under steady vibration, one can extend measurement time and apply an averaging technique to reduce noise for further use such as Fourier transform.

The above experiment demonstrates feasibility of 3D vibration measurement of a measurement point by a single SLDV sequentially placed at three positions. Minimum measurable amplitudes are determined by the sensitivity of a SLDV. Amplitudes of vibration components in a MCS are determined by orientation of the MCS with respect to the direction of shaker excitation. In order to obtain operational deflection shapes or mode shapes of a structure, one can define a series of measurement points on the structure and repeat the same measurement process for each point. A key point is that the single SLDV must measure the exactly same point at each position. A possible solution is to use a camera to record positions of the measurement points, which can be a future research topic. 
Tab. 8 Coordinates of target points in the MCS and corresponding input voltages and initial distances at position 2

\begin{tabular}{cccc}
\hline Coordinates $(\mathrm{mm})$ & $\mathrm{X}$ motor $(\mathrm{V})$ & $\mathrm{Y}$ motor $(\mathrm{V})$ & $r_{0}(\mathrm{~mm})$ \\
\hline$(150,150,0)$ & 3.095 & 1.195 & 2600 \\
$(120,-120,0)$ & 3.375 & -1.835 & 2600 \\
$(-120,-120,0)$ & 6.020 & -1.890 & 2600 \\
$(-150,150,0)$ & 6.392 & 1.250 & 2600 \\
$(-5,35,80)$ & 4.565 & -0.050 & 2520 \\
$(5,-35,150)$ & 4.250 & -0.850 & 2450 \\
\hline
\end{tabular}

Tab. 9 Coordinates of target points in the MCS and corresponding input voltages and initial distances at position 3

\begin{tabular}{cccc}
\hline Coordinates $(\mathrm{mm})$ & $\mathrm{X}$ motor $(\mathrm{V})$ & $\mathrm{Y}$ motor $(\mathrm{V})$ & $r_{0}(\mathrm{~mm})$ \\
\hline$(150,150,0)$ & -3.745 & 2.825 & 2450 \\
$(80,-80,0)$ & -2.950 & 0.080 & 2450 \\
$(-80,-80,0)$ & -1.095 & 0.070 & 2470 \\
$(-150,150,0)$ & -0.300 & 2.715 & 2470 \\
$(-5,35,80)$ & -1.796 & 1.514 & 2380 \\
$(5,-35,150)$ & -1.770 & 0.738 & 2310 \\
\hline
\end{tabular}

Tab. 10 Translation vectors $\mathbf{T}$ of the SLDV at three positions

\begin{tabular}{cccc}
\hline Unit: $\mathrm{mm}$ & $x$ & $y$ & $z$ \\
\hline Position 1 & -1217.4 & -196.3 & 1781.8 \\
Position 2 & -537.4 & -80.9 & 2460.9 \\
Position 3 & 390.8 & -204.7 & 2380.4 \\
\hline
\end{tabular}


Tab. 11 Direction cosine matrices $\mathbf{R}$ of the SLDV at three positions

\begin{tabular}{ccccc}
\hline & & $x^{\prime}$ & $y^{\prime}$ & $z^{\prime}$ \\
\hline \multirow{3}{*}{ Position 1 } & $x$ & -0.6170 & 0.7870 & 0.0041 \\
& $y$ & -0.0404 & -0.0368 & 0.9985 \\
& $z$ & 0.7860 & 0.6159 & 0.0545 \\
\hline \multirow{3}{*}{ Position 2 } & $x$ & -0.3723 & 0.9281 & -0.0001 \\
& $y$ & -0.0466 & -0.0181 & 0.9987 \\
& $z$ & 0.9270 & 0.3718 & 0.0500 \\
\hline \multirow{2}{*}{ Position 3 } & $x$ & 0.2318 & 0.9727 & 0.0083 \\
& $y$ & -0.0492 & 0.0032 & 0.9988 \\
& $z$ & 0.9715 & -0.2319 & 0.0486 \\
\hline
\end{tabular}

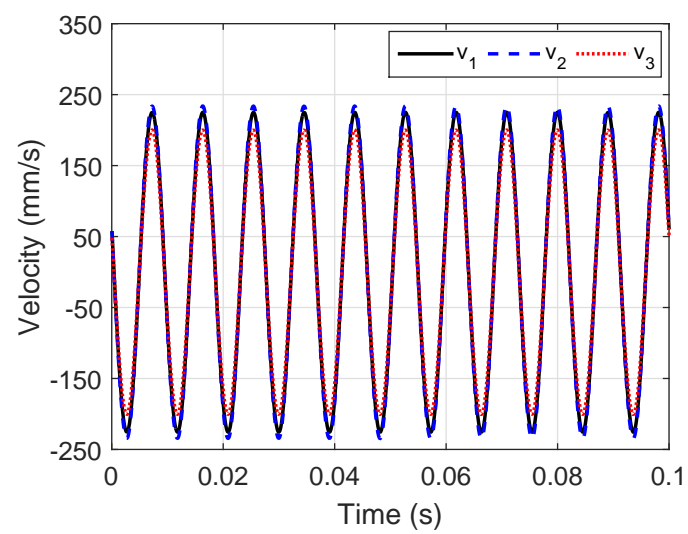

(a)

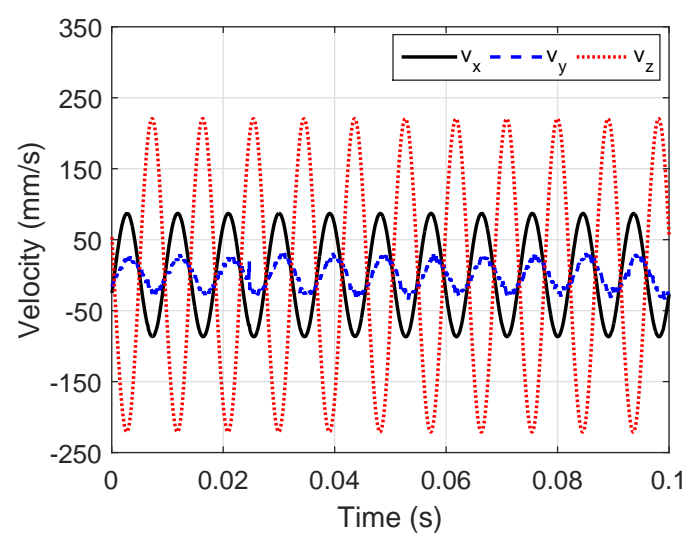

(b)

Fig. 9 (a) Measured velocities by a single SLDV at three positions and (b) velocity components along axes of the MCS calcertated by Eq. (21). 


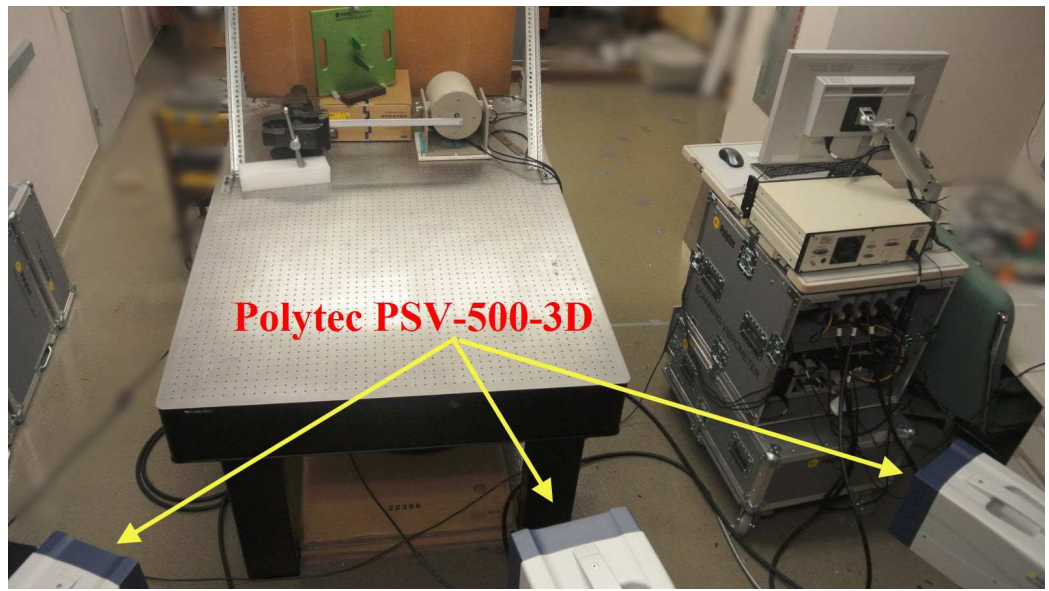

Fig. 10 3D vibration measurement by a commercial Polytec PSV-500-3D.

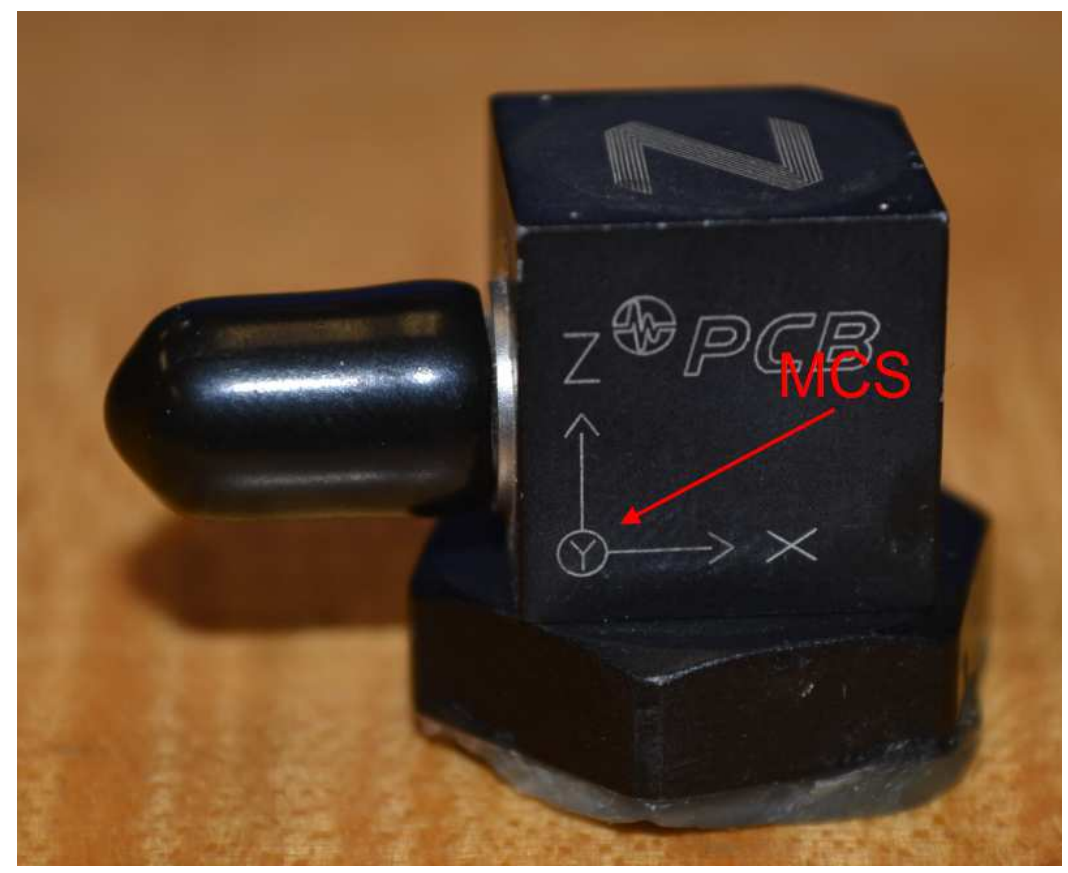

Fig. 11 A tri-axial accelerometer PCB T356A17 with a prescribed MCS. 


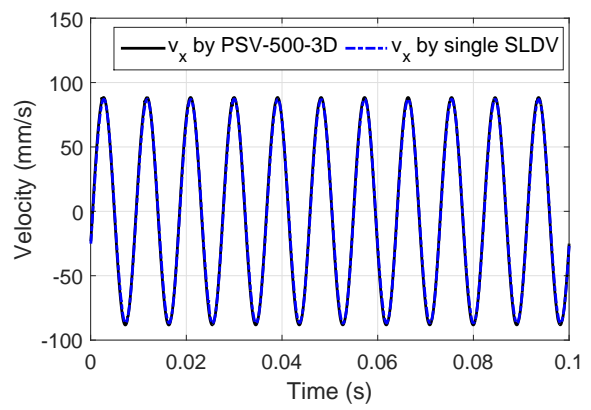

(a)

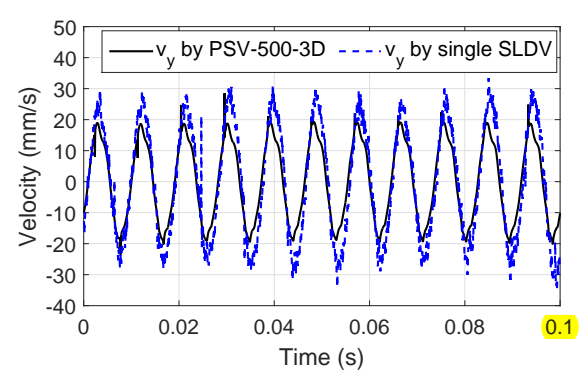

(b)

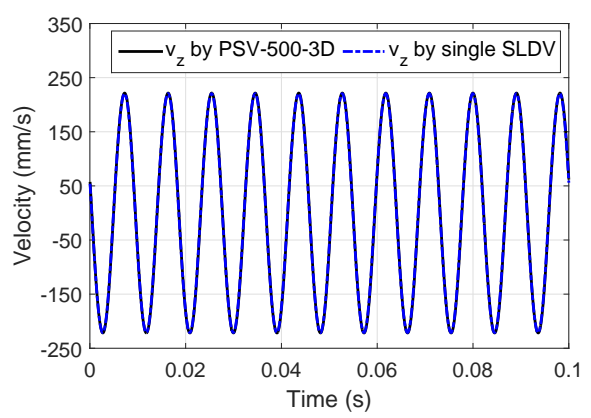

(c)

Fig. 12 Comparison of velocity components from the single SLDV and the PSV500-3D: (a) $x$ component, (b) $y$ component, and (c) $z$ component. 
Tab. 12 Amplitudes of velocity components from the single SLDV and the PSV500-3D

\begin{tabular}{cccc}
\hline Component & Single SLDV $(\mathrm{mm} / \mathrm{s})$ & PSV-500-3D $(\mathrm{mm} / \mathrm{s})$ & Relative error \\
\hline$v_{x}$ & 86.89 & 88.43 & $1.7 \%$ \\
$v_{y}$ & 26.28 & 17.85 & $32.1 \%$ \\
$v_{z}$ & 220.80 & 222.16 & $0.6 \%$ \\
\hline
\end{tabular}

\section{Conclusion}

Investigation of 3D vibration measurement by a single SLDV sequentially placed at three positions is presented in this work. A geometric model of the SLDV is proposed to obtain the relation between the laser beam direction and rotation angles of scan mirrors. An improved method to calculate the pose of a SLDV with respect to a specified MCS is proposed. The proposed method can obtain an orthogonal direction cosine matrix and be applicable to a 2D structure. Effects of the number of target points on the accuracy and stability of the proposed method are investigated and it is shown that the number of target points has little effect on the proposed method. An experiment was conducted to measure 3D vibration of a measurement point on a beam by a single SLDV sequentially placed at three positions. Three measured velocities obtained by the single SLDV were transformed to velocity components along axes of the MCS. The velocity components from the single SLDV were in good agreement with those from a commercial Polytec PSV500-3D, which demonstrates feasibility of 3D vibration measurement by a single SLDV if a structure is under steady vibration.

\section{Acknowledgment}

The authors are grateful for the financial support from the National Science Foundation under Grant numbers CMMI-1229532 and CMMI-1335024.

\section{References}

[1] S. Rothberg, J. Baker, N. A. Halliwell, Laser vibrometry: pseudovibrations, Journal of Sound and Vibration 135 (3) (1989) 516-522.

[2] P. Castellini, G. Revel, E. Tomasini, Laser doppler vibrometry: a review of advances and applications, The Shock and vibration digest 30 (6) (1998) 443-456. 
[3] J. R. Bell, S. Rothberg, Laser vibrometers and contacting transducers, target rotation and six degree-of-freedom vibration: what do we really measure, Journal of Sound and Vibration 237 (2) (2000) 245-261.

[4] P. Castellini, M. Martarelli, E. P. Tomasini, Laser doppler vibrometry: development of advanced solutions answering to technology's needs, Mechanical Systems and Signal Processing 20 (6) (2006) 1265-1285.

[5] A. Stanbridge, D. Ewins, Modal testing using a scanning laser doppler vibrometer, Mechanical systems and signal processing 13 (2) (1999) 255270.

[6] A. B. Stanbridge, M. Martarelli, D. J. Ewins, Measuring area vibration mode shapes with a continuous-scan ldv, Measurement 35 (2) (2004) 181-189.

[7] D. Di Maio, D. Ewins, Continuous scan, a method for performing modal testing using meaningful measurement parameters; part i, Mechanical Systems and Signal Processing 25 (8) (2011) 3027-3042.

[8] P. Giuliani, D. Di Maio, C. Schwingshackl, M. Martarelli, D. Ewins, Six degrees of freedom measurement with continuous scanning laser doppler vibrometer, Mechanical systems and signal processing 38 (2) (2013) 367383.

[9] S. Yang, M. S. Allen, Harmonic transfer function to measure translational and rotational velocities with continuous-scan laser doppler vibrometry, Journal of Vibration and Acoustics 136 (2) (2014) 021025.

[10] P. O'Malley, T. Woods, J. Judge, J. Vignola, Five-axis scanning laser vibrometry for three-dimensional measurements of non-planar surfaces, Measurement Science and Technology 20 (11) (2009) 115901.

[11] L. Mitchell, R. West, A. Wicks, An emerging trend in experimental dynamics: Merging of laser-based three-dimensional structural imaging and modal analysis, Journal of Sound and Vibration 211 (3) (1998) 323333.

[12] C. Vuye, S. Vanlanduit, F. Presezniak, G. Steenackers, P. Guillaume, Optical measurement of the dynamic strain field of a fan blade using a 
3d scanning vibrometer, Optics and Lasers in Engineering 49 (7) (2011) 988-997.

[13] H. Weisbecker, B. Cazzolato, S. Wildy, S. Marburg, J. Codrington, A. Kotousov, Surface strain measurements using a $3 \mathrm{~d}$ scanning laser vibrometer, Experimental mechanics 52 (7) (2012) 805-815.

[14] S. Ihrle, A. Eiber, P. Eberhard, Experimental investigation of the three dimensional vibration of a small lightweight object, Journal of Sound and Vibration 334 (2015) 108-119.

[15] B. Weekes, D. Ewins, Multi-frequency, 3d ods measurement by continuous scan laser doppler vibrometry, Mechanical Systems and Signal Processing 58 (2015) 325-339.

[16] X. Zeng, A. L. Wicks, L. D. Mitchell, Geometrical method for the determination of the position and orientation of a scanning laser doppler vibrometer, Optics and lasers in engineering 25 (4) (1996) 247-264.

[17] Y. Xu, R. Miles, An identification algorithm for directing the measurement point of scanning laser vibrometers, Optics and lasers in engineering 22 (2) (1995) 105-120.

[18] S. J. Rothberg, M. Tirabassi, A universal framework for modelling measured velocity in laser vibrometry with applications, Mechanical Systems and Signal Processing 26 (2012) 141-166.

[19] A. Li, Y. Ding, Y. Bian, L. Liu, Inverse solutions for tilting orthogonal double prisms, Applied optics 53 (17) (2014) 3712-3722.

[20] A. Li, X. Gao, W. Sun, W. Yi, Y. Bian, H. Liu, L. Liu, Inverse solutions for a risley prism scanner with iterative refinement by a forward solution, Applied Optics 54 (33) (2015) 9981-9989.

[21] K. S. Arun, T. S. Huang, S. D. Blostein, Least-squares fitting of two 3-d point sets, Pattern Analysis and Machine Intelligence, IEEE Transactions on (5) (1987) 698-700. 